\title{
7. Herzklappenersatz durch autologe Fascia lata
}

\author{
A. Bernhard, A. Thiede, J. Schatefer, H. K. Müller-Hermelink, \\ K. J. Nordmann, A. C. Yankah, K. Fischer und H. J. SoHwarzkopf \\ Kardiologische Arbeitsgemeinschaft der Chirurgischen Universitätsklinik Kiel, \\ I. Medizinische Universitätsklinik Kiel \\ und Pathologisches Institut der Universität Kiel
}

Von insgesamt 240 Herzklappenersatzoperationen an der Chirurgischen Universitätsklinik Kiel erhielten 40 Patienten autologe Fascia lata-Transplantate. Davon wurde die Aortenklappe in 10 Fällen, die Mitralklappe in 30 Fällen nach der Technik von Ionescu u. Ross ersetzt.

Autologe Fascia lata-Klappen sind auf Grund der zentralen Öffnung und des dadurch möglichen zentralen Blutdurchtritts hämodynamisch prothetischen Klappen deutlich überlegen. Die Thrombosegefährdung der Patienten liegt deutlich niedriger, die Marcumarisierung ist daher höchstens temporär. Die Ergebnisse bei Verwendung dieses Materials in der Aortenklappenregion sind gut. Von bisher 30 Mitralklappenersatzoperationen wurden bei 5 Patienten nach $1 / 2-1$ Jahr Kontrollen der Mitralklappenfunktion durch Herzkatheteruntersuchungen vorgenommen, die bei unseren Patienten im Cineangiogramm eine sich weit öffnende Mitralklappe ergab ohne Zeichen einer Mitralinsuffizienz.

Von 5 Patienten mit Mitralklappentransplantaten, die verstarben oder re-operiert wurden, ergaben licht- und elektronenmikroskopische Untersuchungen, daß die Kollagenfaserbündel nach 6 Monaten noch voll erhalten, während die ursprünglich zwischen den Fasern liegenden Fibrocyten weitgehend geschwunden sind. In der locker strukturierten ventrikelwärts gelegenen Schicht ist die Zellzahl erhöht, bedingt durch eine Zunahme von monocytogenen Fibroblasten, die Zeichen der Phagocytose und Kollagenfasersynthese zeigen. Die Endothelialisierung ist auf Grund der unterschiedlichen Blutstromverhältnisse auf der Vorhofseite meist unterschiedlich und unvollständig nach 6 Monaten, während sie auf der Ventrikelseite immer mehrschichtig und durchgehend ist. Dieses mehrschichtige ,Pseudoendothel“ enthält Zellen mit langen parallel zur Oberfläche verlaufenden Cytoplasmaausläufern, die deutliche Zeichen der Kollagenfasersynthese zeigen. 